\title{
Dendritic Spikes Are Enhanced by Cooperative Network Activity in the Intact Hippocampus
}

\author{
Anita Kamondi, László Acsády, and György Buzsáki \\ Center for Molecular and Behavioral Neuroscience, Rutgers, The State University of New Jersey, Newark, \\ New Jersey 07102
}

\begin{abstract}
In vitro experiments suggest that dendritic fast action potentials may influence the efficacy of concurrently active synapses by enhancing $\mathrm{Ca}^{2+}$ influx into the dendrites. However, the exact circumstances leading to these effects in the intact brain are not known. We have addressed these issues by performing intracellular sharp electrode recordings from morphologically identified sites in the apical dendrites of CA1 pyramidal neurons in vivo while simultaneously monitoring extracellular population activity. The amplitude of spontaneous fast action potentials in dendrites decreased as a function of distance from the soma, suggesting that dendritic propagation of fast action potentials is strongly attenuated in vivo. Whereas the amplitude variability of somatic action potentials was very small, the amplitude of fast spikes varied substantially in distal dendrites. Large-
\end{abstract}

Experiments using extracellular techniques, intracellular recordings with sharp electrodes, and patch-clamp pipettes have led to competing but not necessarily mutually exclusive scenarios regarding the initiation and active propagation of action potentials (Johnston et al., 1996; Yuste and Tank, 1996; Stuart et al., 1997). According to one view, fast $\left(\mathrm{Na}^{+}\right)$action potentials are initiated in the axon, and active dendritic $\mathrm{Na}^{+}$conductances facilitate their back-propagation into the dendritic tree (Turner et al., 1991; Jaffe et al., 1992; Stuart and Sakmann, 1994; Magee and Johnston, 1995; Spruston et al., 1995). Actively back-propagating fast action potentials may affect the efficacy of concurrently active synapses and may influence synaptic plasticity by enhancing $\mathrm{Ca}^{2+}$ influx into the cell (Yuste and Denk, 1995; Christie et al., 1996; Jester et al., 1996; Magee and Johnston 1997; Markram et al., 1997). An alternative view is that strong excitatory synaptic activation may lead to action potentials generated within the dendrites independent of the soma or axon initial segment (Spencer and Kandel, 1961; Llinás and Nicholson, 1971; Wong et al., 1979; Herreras 1990; Turner et al., 1991; Wong and Stuart, 1992; Regehr et al., 1993). Because most experiments in this area of research have been done in the slice preparation, the argument can be made that the amount of depolarization produced by electrical stimulation of presynaptic fibers for the demonstration

Received Sept. 22, 1997; revised Feb. 27, 1998; accepted March 4, 1998.

This work was supported by National Institute of Neurological Diseases and Stroke Grant NS34994, the Human Frontier Science Program, the Whitehall Foundation, and the Soros Foundation. We thank B. Christie, J. Csicsvari, N. L. Golding, D. Johnston, T. Sejnowski, N. Spruston, M. Steriade, K. Svoboda, D. W. Tank, and J. Zackheim for their comments on an earlier version of this manuscript.

Correspondence should be addressed to György Buzsáki, Center for Molecular and Behavioral Neuroscience, Rutgers University, 197 University Avenue, Newark, NJ 07102.

Copyright (ㄷ) 1998 Society for Neuroscience $\quad 0270-6474 / 98 / 183919-10 \$ 05.00 / 0$ amplitude fast spikes in dendrites occurred during population discharges of CA3-CA1 neurons concurrent with field sharp waves. The large-amplitude fast spikes were associated with bursts of smaller-amplitude action potentials and putative $\mathrm{Ca}^{2+}$ spikes. Both current pulse-evoked and spontaneously occurring $\mathrm{Ca}^{2+}$ spikes were always preceded by large-amplitude fast spikes. More spikes were observed in the dendrites during sharp waves than in the soma, suggesting that local dendritic spikes may be generated during this behaviorally relevant population pattern. Because not all dendritic spikes produce somatic action potentials, they may be functionally distinct from action potentials that signal via the axon.

Key words: plasticity; calcium spikes; long-term potentiation; spike propagation; signaling; action potentials of dendritic spike initiation is artificial, and such synchrony never exists under physiological conditions (Mainen et al., 1995; Stuart et al., 1997). Besides factors intrinsic to the neuron, such as channel density and branching pattern of the dendritic tree, various network factors can exert an important effect on dendritic $\mathrm{Na}^{+}$conductances and associated $\mathrm{Ca}^{2+}$ electrogenesis in the in vivo situation, including synaptic inhibition, the extracellular milieu, the discharge frequency of the neuron, and cooperative synchronized depolarization of dendritic segments (Kim et al., 1995; Buzsáki et al., 1996; Miles et al., 1996; Tsubokawa and Ross, 1996). It is therefore important to investigate the rules of dendritic electrogenesis and its modification by physiologically relevant network events in the intact brain (Svoboda et al., 1997).

In the behaving animal, two physiologically antagonistic population patterns are known in the hippocampus: theta waves and sharp waves (Buzsáki et al., 1983). During theta activity, somata of pyramidal cells, in general, are hyperpolarized and rarely fire (Leung and Yim, 1986; Fox, 1989; Soltész and Dechénes, 1993; Ylinen et al., 1995b). In contrast, pyramidal cells are depolarized during sharp waves (SPW) (Ylinen et al., 1995a) and often fire "complex-spike" bursts (Spencer and Kandel, 1961; Ranck, 1973). SPWs are large-amplitude (1-3 mV) aperiodic field potentials (40-100 msec) observed in stratum radiatum of the CA1 region (Buzsáki, 1986, 1989; Suzuki and Smith, 1987) present during awake immobility, consummatory behaviors, and slow-wave sleep. Field SPWs result from the excitation of the dendritic fields of CA1 pyramidal cells and interneurons by their CA3 Schaffer collateral input. The ramp-like depolarization of CA1 neurons induces a dynamic interaction between interneurons and pyramidal cells, the result of which is a short-lived oscillatory field potential (ripple) within stratum pyramidale and a phase-related discharge of the CA1 network at $200 \mathrm{~Hz}$ (Buzsáki et al., 1992; 
Ylinen et al., 1995a). In association with field SPWs, 40,00060,000 cells discharge in concert in the CA3-CA1-subiculumpresubiculum-layer $\mathrm{V}$ entorhinal cortex axis (Chrobak and Buzsáki, 1996). In summary, the SPW burst is the most powerful depolarizing population pattern in the hippocampal formation. We reasoned therefore that if active properties of dendrites are critically influenced by synaptic inputs, then their physiological relevance in the intact brain could be investigated during the occurrence of SPW-associated population bursts.

\section{MATERIALS AND METHODS}

Surgery and recording. Ninety-three rats of the Sprague Dawley strain (250-350 gm) were anesthetized with urethane $(1.3-1.5 \mathrm{gm} / \mathrm{kg})$ and placed in a stereotaxic apparatus. The body temperature of the rat was kept constant by a small-animal thermoregulation device. The scalp was removed, and a small $(1.2 \times 0.8 \mathrm{~mm})$ bone window was drilled above the hippocampus (anteroposterior at $-3.3 \mathrm{~mm}$ from anteromedial edge and lateral at $2.2 \mathrm{~mm}$ from bregma) for extracellular and intracellular recordings. The cisterna magna was opened, and the CSF was drained to decrease pulsation of the brain. A pair of stimulating electrodes $(100 \mu \mathrm{m}$ each, with $0.5 \mathrm{~mm}$ tip separation) was inserted into the left fimbria-fornix (anteroposterior at -1.3, lateral at 1.0, and ventral at 4.1) to stimulate the commissural inputs. An extracellular recording electrode $(20 \mu \mathrm{m}$ insulated tungsten wire) was placed into the CA1 pyramidal layer $0.5-1.0 \mathrm{~mm}$ anterior to the intracellular electrode. Positioning of the recording electrode in the CA1 pyramidal layer was aided by the presence of multipleunit activity and the commissurally evoked responses. After the extracellular and intracellular recording electrodes were inserted into the brain, the bone window was covered by a mixture of paraffin and paraffin oil to prevent drying of the brain and to decrease pulsation.

Micropipettes for intracellular recordings were pulled from $2.0 \mathrm{~mm}$ capillary glass. They were filled with $1 \mathrm{M} \mathrm{K}^{+}$-acetate in $50 \mathrm{~mm}$ Tris buffer, $\mathrm{pH} 7.2$, containing $2 \%$ biocytin for intracellular labeling. In vivo electrode impedances varied from 60 to $100 \mathrm{M} \Omega$. Once stable, intracellular recordings were obtained and evoked, and passive physiological properties of the cell were determined. Only neurons with a resting potential more negative than $-55 \mathrm{mV}$ were included in this study. Because the resting membrane potential fluctuates in vivo, we used the voltmeter readings of the amplifier (Axoclamp-2B; Axon Instruments, Foster City, CA) to obtain an average value integrated over time. Input resistance of the neurons varied between 17 and $67 \mathrm{M} \Omega$. Field activity recorded through the extracellular electrode was filtered between $1 \mathrm{~Hz}$ and $5 \mathrm{kHz}$. The intracellular activity and the extracellular field unit activity were digitized at $10 \mathrm{kHz}$ with 12 bit precision (ISC-16 board; RC Electronics, Santa Barbara, CA). The electrophysiological data were stored on optical disks. In three rats, bicuculline meth-Cl $(5 \mathrm{mg} / \mathrm{kg})$ was injected intraperitoneally after baseline recordings ( $\geq 15 \mathrm{~min})$, and recordings continued for 30-140 min.

Data analysis. The data were analyzed off-line. The extracellular trace was digitally filtered at $120 \mathrm{~dB} /$ octave to separate the fast-ripple waves $(50-200 \mathrm{~Hz})$ and unit activity $(500 \mathrm{~Hz}-5 \mathrm{kHz})$. Cross-correlograms between intracellular spikes and extracellular unit activity were performed using a selected amplitude or pattern of intracellular spikes as reference signals. Extracellular units were detected by amplitude discriminator software. For the amplitude measurements of intracellular fast spikes, the intracellular trace was filtered $(50 \mathrm{~Hz}-5 \mathrm{kHz})$ to eliminate synaptic potentials and slow spikes. These discriminated spikes were used to construct amplitude histograms, interspike interval histograms, and waveform averages using the original unfiltered traces. Spike amplitude, rate of rise, rate of decay, amplitude of spike afterpotential, and halfamplitude width were determined for each recorded neuron.

Histological analysis. After the completion of the physiological data collection, biocytin was injected through a bridge circuit using $500 \mathrm{msec}$ depolarizing pulses at $0.6-2 \mathrm{nA}$ at $1 \mathrm{~Hz}$ for $10-60 \mathrm{~min}$. Neuronal activity was followed throughout the procedure, and the current was reduced if the electrode was blocked and/or the condition of the neuron deteriorated. Two to $12 \mathrm{hr}$ after the injection, the animals were given a urethane overdose and then perfused intracardially with $100 \mathrm{ml}$ of physiological saline followed by $400 \mathrm{ml}$ of $4 \%$ paraformaldehyde and $0.2 \%$ glutaraldehyde dissolved in PBS, $\mathrm{pH}$ 7.2. The brains were then removed and stored in the fixative solution overnight. Sixty- or $100-\mu$ m-thick coronal sections were cut and processed for biocytin labeling (Sik et al., 1995). The labeled neurons were reconstructed with the aid of a drawing tube.
Double- and multiple-labeled neurons were discarded from the analysis. The histological sections were also used to verify the position of the extracellular recording electrodes and the track made by the recording pipette. Both physiological and histological methods were used to localize the tip of the recording electrode. During the experiment the micrometer readings of the microstepper (Inchworm; Burleigh Inc., Fishers, NY) indicated the depth from the pyramidal layer. The pyramidal layer was recognized by the high density of impaled somata. After the withdrawal of the pipette from the dendrite, extracellular averaged evoked responses to commissural stimulation were obtained at $50 \mu \mathrm{m}$ steps from the recording site to the pyramidal layer. The polarity reversal of the extracellular field response at the border of strata radiatum and pyramidale provided an additional landmark for the pyramidal layer. The location of dendritic penetration was determined from the distance between the pyramidal layer and the recording site (measured in a straight line) during the experiment and from the anatomical reconstruction of the electrode track and the dendritic tree of the filled neuron. After the field potential recordings, the pipette was moved up and down several times at a faster speed. This procedure aimed to sever capillaries and to release red blood cells for filling the recording track. In some experiments, the glass pipette was glued to the surface of the brain with cyanoacrylic. The pipette was broken above the glue, and the brain was perfused with the pipette left in situ. During sectioning, the pipette was cut above the corpus callosum by a fine scalpel blade, the upper part of the pipette was removed, and the hippocampus was sectioned with the glass tip remaining in the hippocampus.

\section{RESULTS}

\section{Properties of dendritic action potentials vary with distance from soma}

All recordings in this study were made from biocytin-injected and morphologically identified CA1 pyramidal cells. Double- and multiple-labeled neurons were discarded from the analysis. Stable measurements (30 $\mathrm{min}-4 \mathrm{hr}$; resting membrane potential less than $-55 \mathrm{mV}$ ) were made from 56 dendrites at distances of up to $400 \mu \mathrm{m}$ from the pyramidal layer. Somatic recordings were available from 28 additional neurons. Somatic recordings were characterized by short $(0.98 \pm 0.09 \mathrm{msec}$ half-amplitude width) overshooting action potentials with little amplitude variance, undershooting spike afterhyperpolarization, frequency of spontaneous firing $<2 \mathrm{~Hz}$, and the lack of slow spikes (see below) in response to depolarizing pulses.

During the experiment, the location of the recorded dendrite was estimated from the travel distance of the recording pipette from the pyramidal layer. In addition, after the withdrawal of the pipette from the dendrite the extracellular averaged evoked responses to commissural stimulation were obtained at $50 \mu \mathrm{m}$ steps from the site of dendritic impalement to the pyramidal layer. The polarity reversal of the extracellular field response at the border of strata radiatum and pyramidale provided a clearcut landmark for the pyramidal layer (Andersen et al., 1971). Anatomical verification of the recording track was available in 26 cases. In several instances, the exact location of the impalement could also be verified from the recording track and the reconstructed dendritic tree (Fig. 1A). Occasionally, bulging of the dendrite or the presence of biocytin-filled astrocytic processes further assisted the determination of the location of dendritic impalement.

Recordings from apical dendrites of CA1 pyramidal cells replicated several features of dendritic action potentials described in vitro (Wong et al., 1979; Wong and Stuart, 1992; Spruston et al., 1995; Tsubokawa and Ross, 1996). The amplitude of fast action potentials was always smaller than that recorded from the soma (Fig. 2A). Depolarizing pulses in dendrites evoked a train of action potentials with progressively decrementing amplitude. The recordings shown in Figure 1 were obtained from the distal part of a primary dendritic shaft in the outer third of stratum radia- 

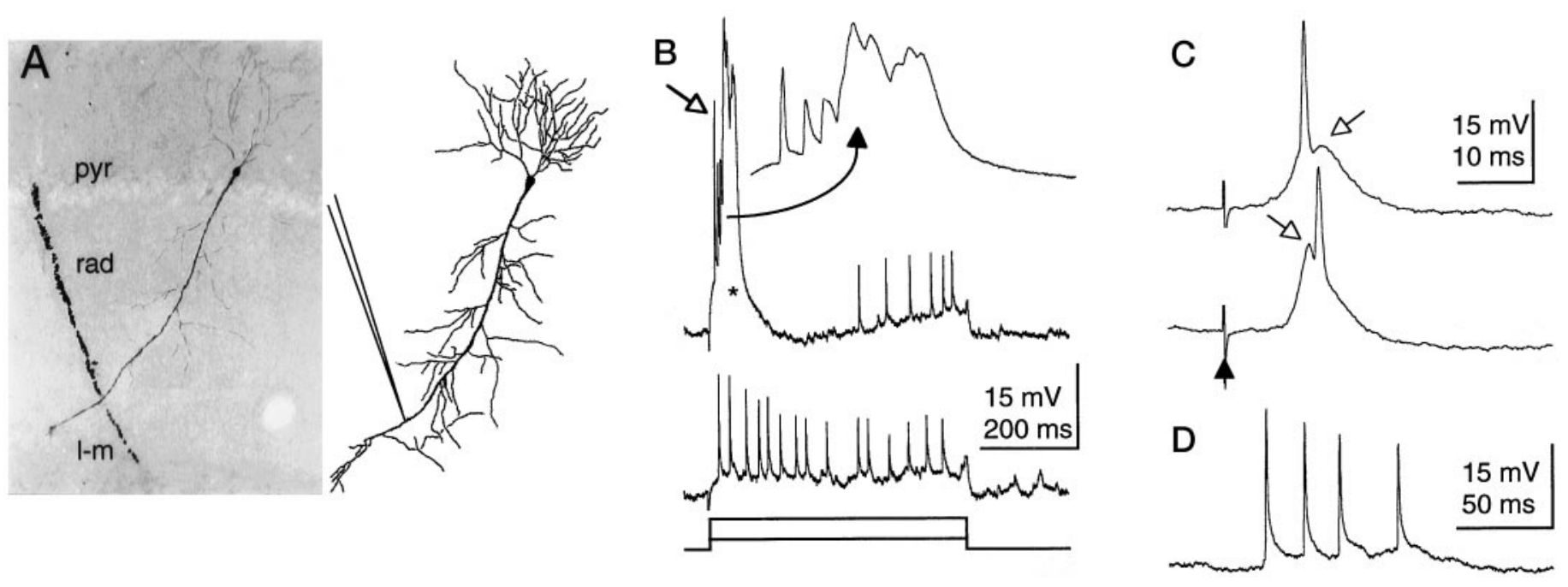

Figure 1. Properties of evoked and spontaneous dendritic events. A, Photograph of the electrode track and the biocytin-filled neuron and its camera lucida reconstruction. Recording was made from the principal apical shaft at the border of strata radiatum ( $\mathrm{rad})$ and lacunosum-moleculare $(l-m)$. The electrode was moved beyond the dendrite during the experiment. The electrode track is filled with red blood cells. pyr, Pyramidal layer. $B$, Current step-induced responses (0.3 and $1.0 \mathrm{nA}$ ). Note amplitude decrement of fast spikes (bottom trace). Note also large fast spike (arrow), slow spike (asterisk), and transient cessation of fast spikes (top trace). Inset, Temporal detail of the fast and slow spikes. $C$, Responses to commissural input stimulation (arrow). Traces were evoked using the same stimulus intensity. Note that the spike can precede or follow the peak depolarization (arrows). $D$, Spontaneous spike burst with decrementing amplitude spikes.
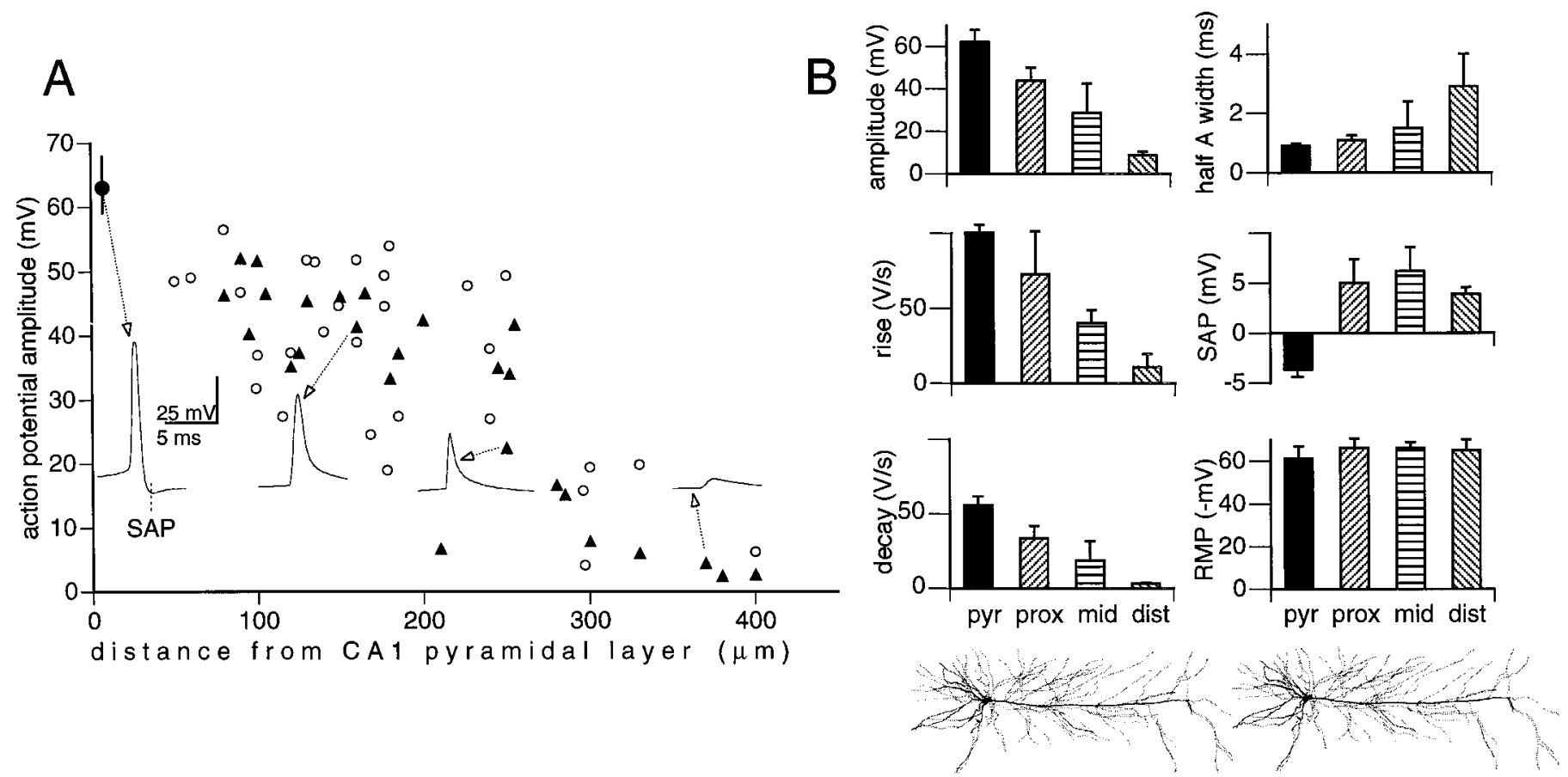

Figure 2. Properties of spontaneous dendritic action potentials in CA1 pyramidal cells in vivo. A, Amplitude of action potentials (measured from the inflection point to the peak) as a function of the distance from the cell body. Each point is an average of at least 50 spontaneous single spikes. The average somatic spike amplitude is indicated by a single point (mean $\pm \mathrm{SD} ; n=25)$. Insets, Examples of averaged spikes $(n=50)$. Filled triangles, Anatomically verified dendritic locations; open circles, dendritic recording sites are estimated from the distance from the cell body layer during the recording session. $B$, Spike amplitude, rate of rise, rate of decay, half-amplitude width $($ half $A)$, fast spike afterpotential $(S A P)$, and resting membrane potential $(R M P)$ in the soma ( pyr) and at dendritic sites (mean $\pm \mathrm{SD}$ ). The stratum radiatum was arbitrarily divided into three equal layers: proximal ( prox), middle (mid), and distal (dist).

tum. Low-intensity current steps $(<0.4 \mathrm{nA})$ induced a moderate spike frequency adaptation and amplitude decrement of the action potentials. Larger current steps induced a more complex pattern associated with three additional changes. First, a largeamplitude slow spike, likely reflecting high-threshold $\mathrm{Ca}^{2+}$ cur- rents (Wong et al., 1979; Wong and Stuart, 1992; Magee and Johnston, 1995), was evoked. Second, the slow spikes were associated with a burst of fast spikes, one of which was often much larger in amplitude (Fig. 1B, arrow) than those evoked by smaller currents. Third, large-amplitude slow spikes were followed by 

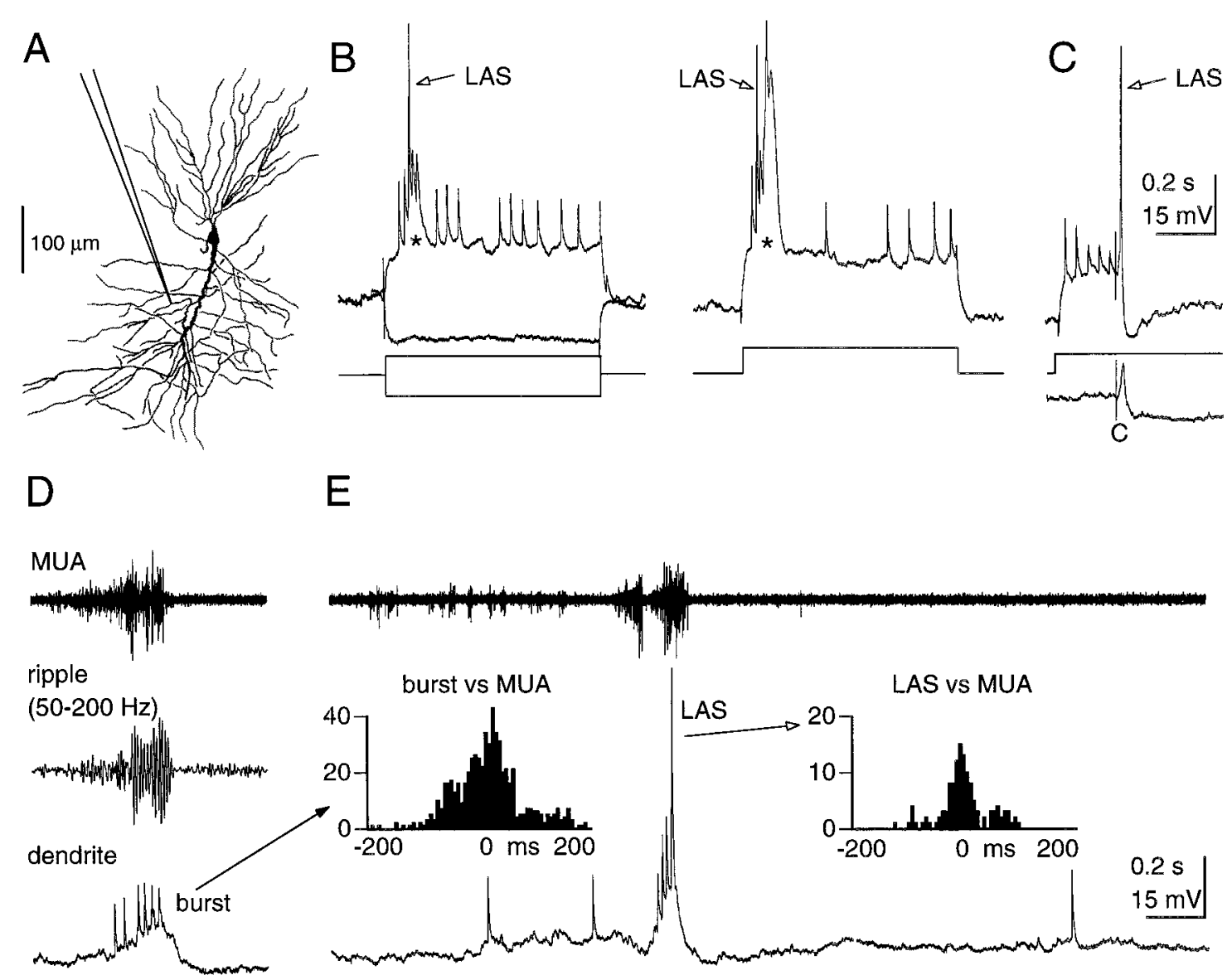

Figure 3. Sharp wave burst-induced amplitude enhancement of fast spikes. $A$, Reconstructed dendritic tree. The micropipette points to the anatomically verified penetrated dendrite. $B$, Responses to hyperpolarizing $(-0.5 \mathrm{nA})$ and depolarizing (left, $0.5 \mathrm{nA}$; right, $0.6 \mathrm{nA})$ current steps. Arrow, Large-amplitude fast spike $(L A S)$; asterisk, putative calcium spike. $C$, Responses to commissural $(c)$ stimulation. Note large-amplitude-evoked fast spike (arrow) and absence of spike (bottom trace) with and without concurrent direct depolarization of the dendrite, respectively. The same stimulus intensity was used in both cases. $D, E$, Relationship between extracellularly recorded multiple unit activity $(M U A)$ and field ripples from CA1 pyramidal layer and intradendritic activity (dendrite). There is a $45 \mathrm{sec}$ gap between traces in $D$ and $E$. Cross-correlogram (burst vs $M U A$ ) between intradendritic bursts as defined by repeating spikes at $<10 \mathrm{msec}$, interspike intervals, and extracellular MUA activity illustrates that the incidence of intradendritic bursts was highest during ripple-related MUA. E, left inset, Large-amplitude fast spikes were present exclusively during MUA bursts. Note that the slow potential associated with the large fast spike is larger than the stimulation-evoked EPSP shown in C. Right inset, Cross-correlogram between large-amplitude ( $>30$ $\mathrm{mV})$ spikes and MUA activity $(L A S v s M A)$. Ordinate, Number of units per bin.

suppression of fast spikes, likely attributable to activation of $\mathrm{Ca}^{2+}$-mediated increase of $\mathrm{K}^{+}$conductance (Fig. 1) (Hotson and Prince, 1980; Schwartzkroin and Stafstrom, 1980). Synaptically evoked postsynaptic potentials in dendrites were of larger amplitude, and the latency of evoked action potential varied more than in the soma. Action potentials could occur before or after the peak of the evoked EPSP (Fig. 1C).

In addition to the above differences, dendritic and somatic fast action potentials were different in several more respects. The amplitude of spontaneously occurring single action potentials decreased as a function of the distance from the soma (Fig. 2). Several other parameters of spontaneously occurring spikes, including the rate of rise and decay, the width at half-amplitude, and the spike afterpotential, also correlated with the location of the dendritic recording (Fig. $2 B$ ). The undershooting spike afterhyperpolarization observed in somatic recordings was absent or, in fact, was depolarizing in the dendrite. The resting membrane potential was slightly but significantly more hyperpolarized in the dendrites $(-65.2 \pm 2.2 \mathrm{mV})$ than in the soma $(-61.2 \pm 1.9 \mathrm{mV}$; $t=3.26 ; p<0.002)$. However, the resting membrane potential did not change with distance from the soma.

The amplitude of spontaneous spikes in dendrites was rather constant from the soma up to $\sim 250 \mu \mathrm{m}$ from the somatic layer (Fig. 2A). This was followed by a step-wise amplitude decrement in the distal third of stratum radiatum. Regression lines fitted to the proximal-middle group $(<280 \mu \mathrm{m})$ and distal group intercepted the $y$-axis at 55 and $48 \mathrm{mV}$, respectively. However, the slopes of the linear regression lines were significantly different $\left(F^{(6,30)} ; p<0.015\right)$. In only one case was the spike amplitude $<15$ $\mathrm{mV}$ in the proximal two-thirds of stratum radiatum. In this single case (Fig. 2, triangle at $220 \mu \mathrm{m}$ ), the recordings were made from an anatomically verified second-order branch.

\section{Network influence on dendritic spikes}

A striking difference between somatic-proximal dendritic and distal dendritic recordings was the large range of spike amplitudes in distal dendrites. Although recordings at distal locations typically revealed small action $(<15 \mathrm{mV})$ potentials, large-amplitude 
A

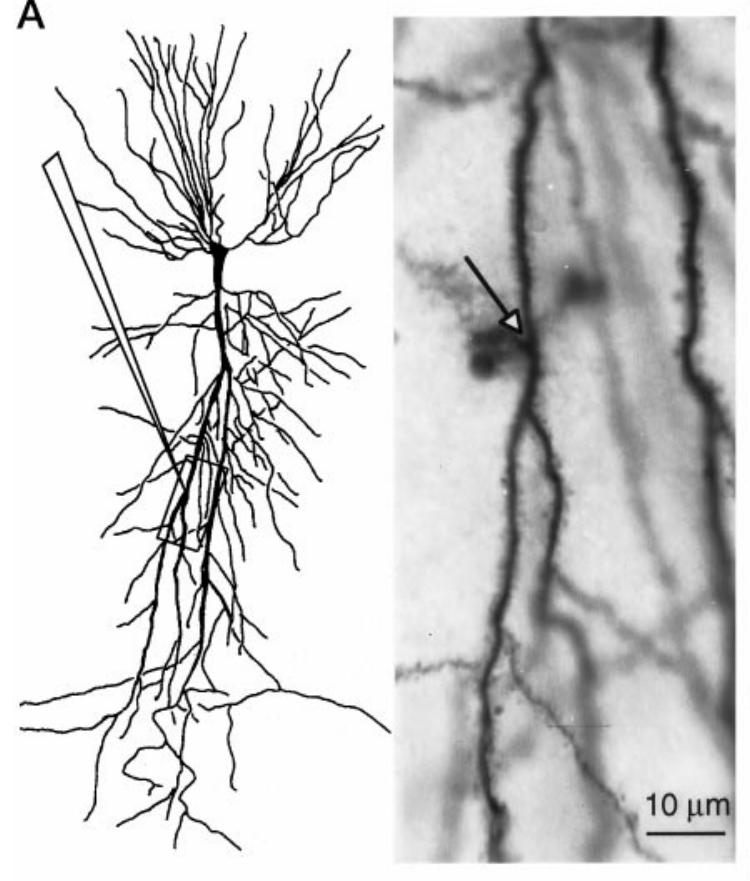

B
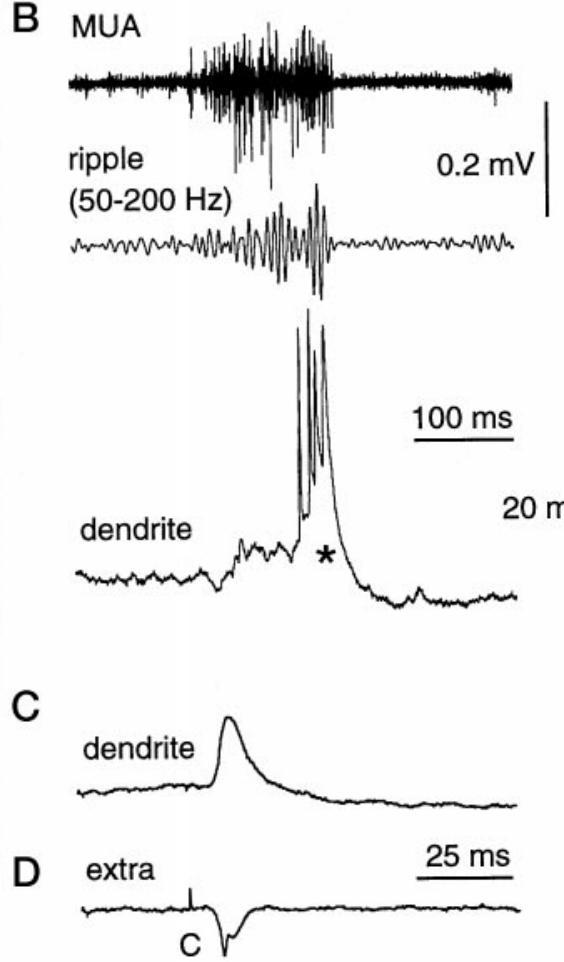

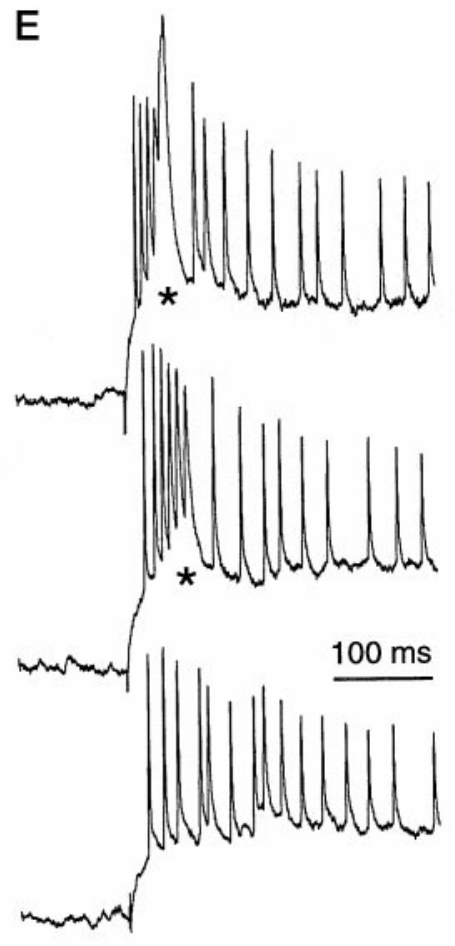

Figure 4. Recording from a first-order dendrite in the middle third of stratum radiatum. A, Reconstructed dendritic tree. The arrow (enlargement of the boxed area) shows a labeled astrocytic process at the site of electrode penetration. $B$, Relationship between extracellularly recorded multiple unit activity $(M U A)$ and field ripples from CA1 pyramidal layer and intradendritic activity. $C, D$, Intradendritic (dendrite)-evoked potential in response to commissural $(c)$ stimulation and extracellular response (extra) after the pipette was withdrawn from the dendrite. E. Current step-induced responses (0.4, 0.8 , and $1.0 \mathrm{nA})$. Asterisks, Putative calcium spikes. Note that the commissurally evoked response $(C)$ is smaller than the large depolarization associated with the sharp wave burst in $B$. Note also the similarity of the spontaneous and current-induced bursts.

$(20-50 \mathrm{mV})$ spontaneous spikes were also observed as part of a burst (Fig. 3). When action potentials were evoked by intradendritic current pulses, the amplitude of the first few evoked spikes was often larger than that of the spontaneously occurring action potentials. Spikes evoked by suprathreshold afferent stimulation were also larger in amplitude than the spontaneously occurring spikes. This was in sharp contrast to somatic recordings, because the amplitude of evoked spikes in the soma was always $10-30 \%$ smaller than that of the spontaneously occurring action potentials, likely attributable to a shunting effect of IPSPs mediated by the feed-forward activation of basket cells (Sik et al., 1995; Miles et al., 1996). Subthreshold afferent stimulation combined with intradendritic current-induced depolarization often evoked largeamplitude spikes (Fig. 3C). These findings indicated that local regeneration of fast spikes can be enhanced by depolarization of the dendrite.

After studying dendritic events in response to current injections and electrically evoked synaptic responses, we questioned whether the magnitude of dendritic depolarization required for these events is ever achieved under physiological conditions by comparing the intracellular spike events with the extracellularly recorded field and multiple unit activity. Field activity was characterized by either rhythmic theta waves $(2-6 \mathrm{~Hz})$ or irregularly occurring sharp wave events (SPW) associated with a fast field oscillation in the pyramidal layer (ripple) and population discharge of a large number of neurons (Ylinen et al., 1995a). Dendritic action potential bursts occurred most consistently dur- ing SPWs, although they were occasionally observed during theta activity and between the irregularly occurring SPWs. All recordings illustrated in the present work were obtained during SPW (nontheta) state. The relationship between intracellular events and the extracellularly recorded multiple unit activity (MUA) was quantified by cross-correlating these events. As was the case with somatically recorded action potentials (Ylinen et al., 1995), SPWassociated field ripples and MUA correlated with dendritic bursts of action potentials (Fig. 3E, left inset). A burst of action potentials was defined as repeating spikes at $<10 \mathrm{msec}$ interspike intervals for these calculations. In each animal with a sufficient number of SPW bursts $(>10)$ for the construction of crosscorrelograms $(n=17)$, the large peaks in the cross-correlograms indicated that intradendritic bursts occurred preferentially during the SPW-associated population events.

Similar to the current injection-induced events, largeamplitude spikes never occurred in isolation but were always part of a dendritic burst. In distal dendrites the amplitude of the small and large spikes could easily be distinguished. To study the role of network activity in the generation of the large-amplitude events, the large-amplitude spikes were cross-correlated with the simultaneously recorded MUA (Fig. 3E, right inset). The peaks in the cross-correlograms indicated that large-amplitude spikes occurred preferentially during the SPW-associated population events ( $n=6$ distal dendrites). In four cases, spontaneous largeamplitude spikes were present exclusively during SPW bursts.

SPW-associated large, fast spikes, similar to current pulse- 

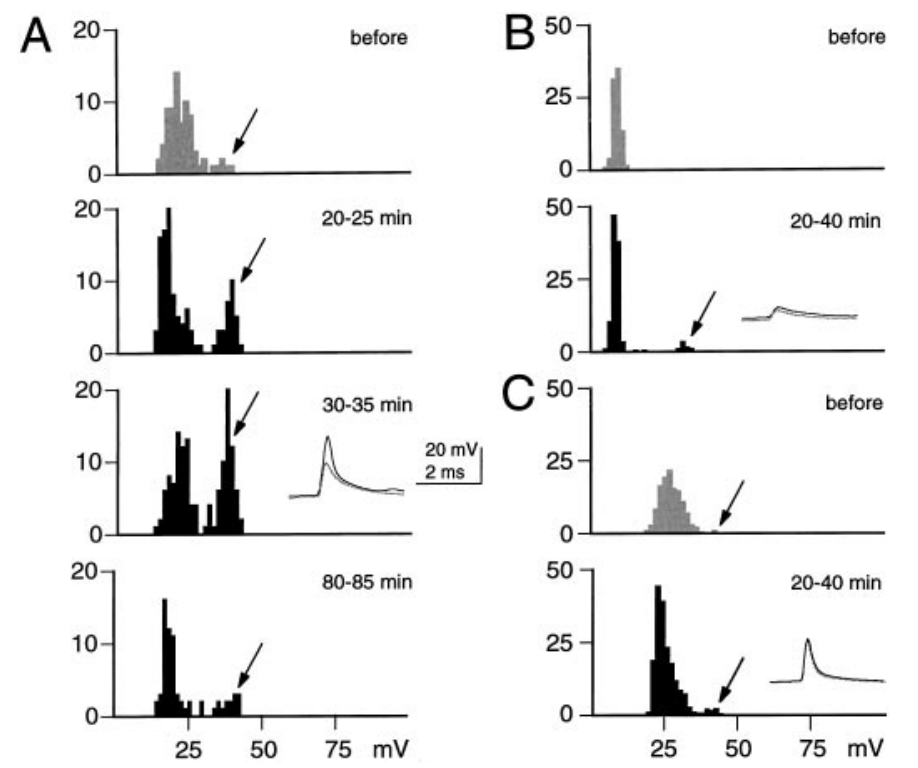

Figure 5. Augmentation of fast spike amplitude by attenuating GABAergic inhibition. Amplitude histograms of action potentials in three dendrites $(A-C)$ before (gray) and after (black) bicuculline administration (5 $\mathrm{mg} / \mathrm{kg}$, i.p.). Note bimodal distribution of dendritic spike amplitude in all three cells. Arrows indicate large-amplitude spikes. Note also the increased incidence of large-amplitude spikes after drug administration. Insets, Action potential averages before (gray) and after (black) bicuculline in the indicated time window. The postdrug average is slightly shifted upward in $B$. $A$ and $C$ are midapical dendrites. $C$ (also shown in Fig. 6) is recorded from a distal dendrite.

induced events, arose from wide depolarizing potentials. These wide depolarizing potentials probably reflected a combination of synaptically induced postsynaptic potentials and $\mathrm{Ca}^{2+}$ spikes, because their amplitude was often larger than that of the commissurally evoked depolarization (Figs. $3 C, E, 4)$. In addition, the rate of decay of these wide events was faster than their rate of rise, similar to the current step-induced $\mathrm{Ca}^{2+}$ spikes. Putative calcium spikes occurred very rarely $(<0.2 / \mathrm{min})$ at the resting potential, but steady depolarization of the dendrite $(<10 \mathrm{mV})$ significantly increased the incidence of putative calcium spikes and revealed them in neurons in which no such events were observed at rest $(50-350 \% ; t=2.45 ; p<0.05 ; n=12)$. These observations indicate that at least part of the SPW-associated large depolarizing events reflect $\mathrm{Ca}^{2+}$ spikes. In an attempt to increase the incidence of large-amplitude fast spikes and associated putative $\mathrm{Ca}^{2+}$ spikes, the frequency of sharp wave bursts was increased by reducing inhibition. Systemic injection of the $\mathrm{GABA}_{\mathrm{A}}$ receptor blocker bicuculline $(5 \mathrm{mg} / \mathrm{kg}$, i.p.) increased the amplitude of the commissurally evoked population spike and the incidence of SPW bursts (Buzsáki, 1986). Parallel with these network changes, the incidence of large-amplitude spikes also increased (Fig. 5). The distribution of spike amplitude was bimodal, and bicuculline disproportionately increased the number of largeamplitude spikes (Fig. 5, arrows). Similar to the predrug condition, the large-amplitude fast spikes occurred most often in association with SPWs (Fig. 6). The large-amplitude fast spikes, in turn, were associated with putative $\mathrm{Ca}^{2+}$ spikes. Averages triggered by the large-amplitude fast spikes $(n=3$ rats with bicuculline treatment; $n=4$ rats with no drug treatment) revealed that fast spikes preceded the peaks of the putative $\mathrm{Ca}^{2+}$ spikes (Figs. $6 D, 7 B 2)$. In four control rats, only the vehicle $(0.9 \% \mathrm{NaCl})$ was injected after 20-60 min of dendrite impalement. This injection procedure had no effect on the electrophysiological properties of the recorded neurons.

\section{Action potentials may be initiated at multiple sites}

SPW bursts were typically associated with a single spike in the soma, and the number of somatic spikes during bursts never exceeded four (mean $\pm \mathrm{SD}=1.2 \pm 0.21 ; n=9$; Fig. $7 A$ ). In contrast, SPW events often evoked multiple spike bursts with as many as seven fast action potentials in dendrites (mean $\pm \mathrm{SD}=$ $3.3 \pm 0.65 ; n=16 ; t=4.56 ; p<0.001)$. In addition, comparison of spike autocorrelograms revealed significantly longer refractory periods of somatic spikes $(6.9 \pm 1.1 \mathrm{msec})$ than of dendritic action potentials $(2.7 \pm 0.52 \mathrm{msec} ; t=2.87 ; p<0.01)$. In distal dendritic recordings the refractory period could be as short as the action potential when small- and large-amplitude spikes were all considered (Fig. $7 A, B$ ). When only large-amplitude spikes recorded in distal dendrites were considered, interspike intervals were long (Fig. $7 B$ ). These observations are compatible with the view that action potentials can emanate from multiple locations.

\section{DISCUSSION}

Our results demonstrate that (1) the propagation of fast action potentials in dendrites is strongly suppressed in vivo; (2) largeamplitude dendritic fast spikes occur during SPW bursts; (3) large-amplitude dendritic fast spikes are associated with spike bursts and putative $\mathrm{Ca}^{2+}$ spikes; and (4) fast spikes may be initiated in dendrites and may not invade the soma.

\section{Spike amplitude attenuation in dendrites}

Our in vivo observations of the amplitude decrease of fast spikes with distance from the soma revealed similarities to previous in vitro results (Spruston et al., 1995; Tsubokawa and Ross, 1996; Magee and Johnston, 1997; Jung et al., 1997). However, in in vitro measurements the first spike of the current step-induced train showed little amplitude decrement with distance from the soma, and only subsequent action potentials were attenuated (Callaway and Ross, 1995; Spruston et al., 1995; Tsubokawa and Ross, 1996). This is in contrast to the in vivo situation, because the majority of spontaneous spikes in dendrites were much smaller than in the soma. Thus, the somadendritic amplitude gradient of spontaneous single action potentials in vivo was similar to the later action potentials in evoked trains in vitro. These findings therefore indicate that dendritic propagation of fast action potentials in the intact hippocampus is under a strong tonic suppression. Such suppression of dendritic activity may be brought about by inhibitory interneurons innervating the dendrites of pyramidal cells (Buzsáki et al., 1996; Miles et al., 1996; Tsubokawa and Ross, 1996).

Simultaneous patch-clamp recordings from the soma and different-caliber dendrites showed a step-like reduction in the imaged $\mathrm{Ca}^{2+}$ signal at dendritic branch points (Spruston et al., 1995). Our observations on spontaneously occurring spikes are compatible with the suggestion that dendritic branch points play a critical role in the regulation of action potential back propagation. Although spike amplitudes were somewhat smaller in the middle third of stratum radiatum than in the proximal third, a large step-wise amplitude reduction was evident in the distal third. Most of our anatomically verified impalements were made in the main apical shaft, which in CA1 pyramidal neurons extends up to the distal third of stratum radiatum (Ishizuka et al., 1995). In only one rat was the dendritic spike amplitude $<15 \mathrm{mV}$ in the 

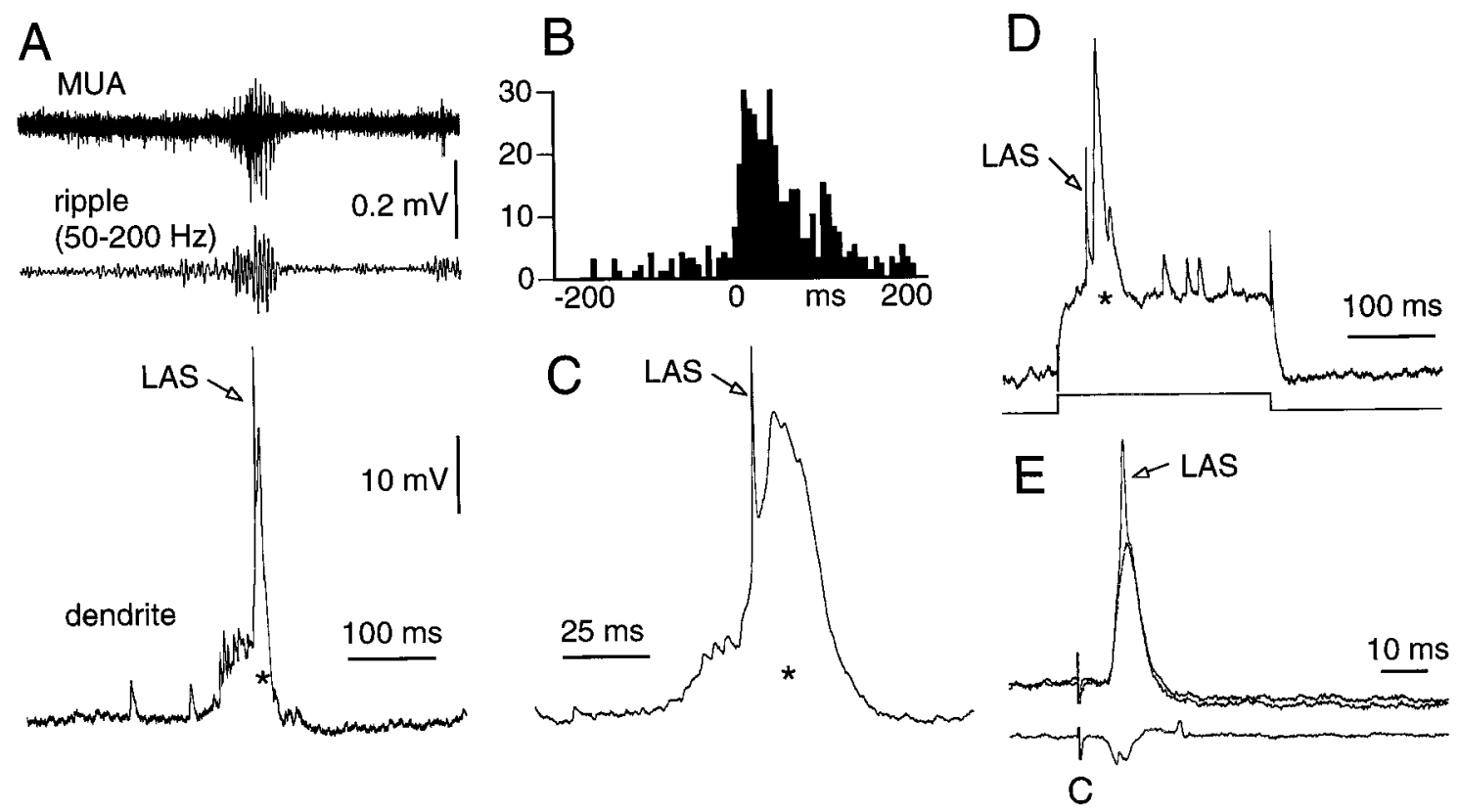

Figure 6. Augmentation of spike amplitude by attenuating GABAergic inhibition. Recordings from a distal dendrite $350 \mu \mathrm{m}$ from the soma. $A$, Relationship between extracellularly recorded multiple unit activity (MUA) and field ripple from CA1 pyramidal layer and intradendritic activity 11 min after drug injection. $B$, Cross-correlation between large-amplitude fast spikes ( $L A S$ in $A ;>20 \mathrm{mV})$ and MUA. $C$, Large-amplitude fast spike-triggered average of intradendritic events $(n=8) . D$, Current step $(0.5 \mathrm{nA})$-induced response before drug administration. $E$, Two superimposed evoked potentials in response to commissural $(c)$ stimulation at threshold intensity 110 min after drug administration. Only one of the stimuli evoked a spike. Bottom trace, Extracellular response after the pipette was withdrawn from the dendrite. Arrows, Large-amplitude spikes; asterisks, putative calcium spikes.

middle third of stratum radiatum. In this case, the recording was made from a histologically verified second-order thin branch. Because all branches in the outer third of the stratum radiatum and in stratum lacunosum-moleculare are second- or higher-order branches, the small-amplitude spikes recorded from dendrites in this layer were thin dendritic segments. These observations suggest that it is the branching order, and consequently the increased density of A-type $\mathrm{K}^{+}$channels (Hoffman et al., 1997), that may determine the degree of spike attenuation in the dendritic tree rather than the metric distance from the soma.

\section{Sharp wave bursts facilitate dendritic electrogenesis}

Of all known physiological patterns, SPWs are associated with the most powerful depolarization of pyramidal cells. Although most interneurons also show intense spiking, their population synchrony is severalfold less than that of the pyramidal cells (Csicsvari et al., 1997). Therefore, during SPWs there is a net increase of synaptic excitation. Dendritic recordings revealed three correlated events during SPWs: bursts of spikes, large-amplitude fast spikes, and wide putative $\mathrm{Ca}^{2+}$ spikes. Large-amplitude action potentials typically occurred with small spike bursts, and large fast spikes were associated with putative $\mathrm{Ca}^{2+}$ spikes. In fact, large-amplitude fast spikes always preceded the peak of the $\mathrm{Ca}^{2+}$ spikes, suggesting that the depolarization produced by the enhanced-amplitude spike is a necessary condition for the activation of high-threshold $\mathrm{Ca}^{2+}$ channels. Although largeamplitude fast spikes in distal dendrites occurred almost exclusively during SPWs, only a portion of SPWs (12\%) was associated with large amplitude spikes. Spontaneous $\mathrm{Ca}^{2+}$ spikes occurred even more rarely $(<0.2 / \mathrm{min})$. The discrepancy between the relative regularity of the population event (SPW) and the rare events in the dendrites may be explained by assuming that coincident presynaptic activity only rarely converges on the recorded dendritic segment. In any case, the amplitude variability of dendritic spikes suggests that action potentials are under the control of local factors. Enhancement of fast spike amplitude may reflect facilitated back-propagation of the action potential from the soma to the recorded dendrite or a locally generated event. Indeed, the average size of action potential decreased progressively toward the distal dendrites. Nevertheless, when convergent network excitation was powerful enough during SPW bursts, large-amplitude spikes were also present in distal dendrites. The enhancement of fast spike amplitude may reflect facilitated back-propagation of the action potential from the soma to the recorded dendrite or a locally generated event. The observation that current-induced intradendritic depolarization enhanced spike amplitude in response to synaptic activation indicates that the magnitude of local membrane depolarization and the speed at which these changes take place are both critical factors.

A general requirement of synaptic plasticity is that afferent activity is present during periods of large postsynaptic depolarization and intradendritic increase of $\mathrm{Ca}^{2+}$ (Bliss and Collingridge, 1993). Recent fluorescent imaging studies indicate the possible involvement of voltage-gated $\mathrm{Ca}^{2+}$ channels in synaptic plasticity (Jaffe et al., 1992; Miyakawa et al., 1992; Regehr and Tank, 1992). Pairing of afferent stimulation with dendritic action potentials can induce a robust $\mathrm{Ca}^{2+}$ influx and long-term modification of the active synapses (Christie et al., 1996; Magee and Johnston 1997; Markram et al., 1997). In light of these experiments, the present observations indicate that in the intact hippocampus these conditions may be present during physiological SPW bursts. During the time window of the SPW, intracellular $\mathrm{Ca}^{2+}$ may be increased by several cooperative mechanisms. 

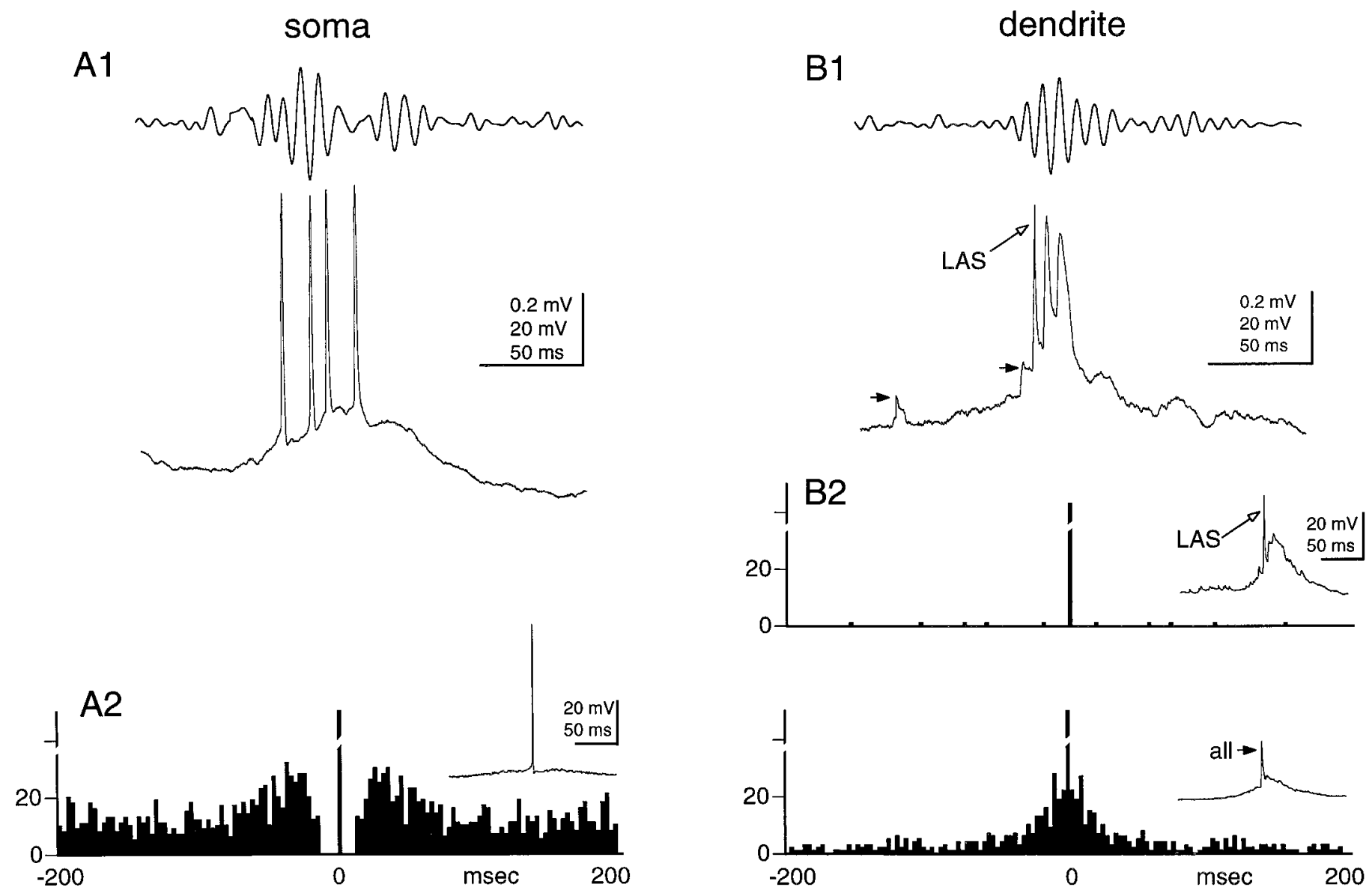

Figure 7. Fast spikes may be initiated at multiple locations. $A 1$, Simultaneous recording of field ripple and intracellular response from soma. Note burst of fast spikes with similar amplitude. A2, Autocorrelogram of fast spikes. Note refractory period of $>8$ msec. Inset, Averaged somatic spike. B1, Simultaneous recording of field ripple and intracellular response from dendrite. Note the presence of small-amplitude ( filled arrow) and large-amplitude (LAS, open arrow) fast spikes. B2, Autocorrelogram of fast large spikes (top) and all spikes (bottom). Note lack of a refractory period when all spikes were included. Inset, Averaged waveforms triggered by large amplitude fast spikes $(L A S)$ and all spikes (all).

Bursts of $\mathrm{Na}^{+}$-dependent action potentials may lead to $\mathrm{Ca}^{2+}$ influx through voltage-gated $\mathrm{Ca}^{2+}$ channels (Jaffe et al., 1992; Miyakawa et al., 1992; Christie et al., 1995; Svoboda et al., 1997). Wide $\mathrm{Ca}^{2+}$ spikes may further increase intracellular $\mathrm{Ca}^{2+}$ levels (Jaffe et al., 1992). The temporal overlap of presynaptic activity and postsynaptic spiking during SPW may result in supralinear summation of $\mathrm{Ca}^{2+}$ signals in dendritic spines (Yuste and Denk, 1995; Hoffman et al., 1997; Magee and Johnston, 1997). Finally, the SPW-associated dendritic depolarization and postsynaptic spiking together or separately may facilitate opening of NMDA channels, thus providing another route for $\mathrm{Ca}^{2+}$ influx. It has yet to be established whether these various mechanisms are present simultaneously during SPWs, and future research will disclose the mechanism of their cooperativeness.

\section{Fast action potentials may be generated in dendrites}

One of the most intriguing and controversial issues regarding dendritic function is whether action potentials have a fixed site of generation in the axon initial segment or whether they can be initiated at multiple locations (Shepherd and Brayton, 1987; Llinás and Nicholson, 1971; Segev and Rall, 1988; Jaslove, 1992; Softky, 1994; Traub et al., 1994). The initiation of $\mathrm{Na}^{+}$action potentials in the axon initial segment or the axon and their active back propagation to the dendritic tree have been directly verified under some conditions. The initiation of $\mathrm{Na}^{+}$action potentials in dendrites is more controversial (Stuart et al., 1997). Dendritically generated spikes in cortical pyramidal cells have been observed by extracellular field recordings and by intracellular recordings with sharp electrodes and patch pipettes (Spencer and Kandel, 1961; Wong et al., 1979; Herreras, 1990; Turner et al., 1991; Wong and Stuart, 1992; Regehr et al., 1993). An important argument against the physiological relevance of these observations is that all experiments used an excessive synchrony of presynaptic fibers induced by electrical stimulation (Mainen et al., 1995; Stuart et al., 1997). Although many of the action potentials recorded in dendrites in vivo likely reflect action potentials initiated in the soma and back-propagating into the dendrites, two observations indicate that action potentials can also be initiated at dendritic locations. First, more spikes were observed during SPW bursts in dendritic recordings than in somatic recordings. The additional dendritic spikes must be generated in the dendrites but must not propagate reliably enough to trigger action potentials at the soma. Second, when small- and large-amplitude spikes were considered, the interspike intervals in dendritic recordings were shorter than expected on the basis of spike refractoriness. The refractory period for dendritically initiated spikes likely extends to some 
distance over which the spike propagates actively, but spikes may be generated at other dendritic locations while one dendritic spike initiation zone is refractory. The most parsimonious explanation of our findings is that action potentials emanated from multiple sites. In support of our in vivo observations, simultaneous recordings from the soma and dendrites of CA1 pyramidal cells in vitro indicate that focally triggered spikes in dendrites often fail to invade the soma (N. L. Golding and N. Spruston, personal communication). These findings therefore indicate that the function of action potentials confined to the dendrites is fundamentally different from somatic action potentials that signal via the axon. One of these functions may be to trigger local $\mathrm{Ca}^{2+}$ spikes and modify synaptic strength.

\section{REFERENCES}

Andersen P, Bliss TVP, Skrede KK (1971) Lamellar organization of hippocampal excitatory pathways. Exp Brain Res 13:222-238.

Bliss TVP, Collingridge GL (1993) A synaptic model of memory: longterm potentiation in the hippocampus. Nature 361:31-39.

Buzsáki G (1986) Hippocampal sharp waves: their origin and significance. Brain Res 398:242-252.

Buzsáki G (1989) Two-stage model of memory trace formation: a role for "noisy" brain states. Neuroscience 31:551-570.

Buzsáki G, Leung LS, Vanderwolf CH (1983) Cellular basis of hippocampal EEG in the behaving rat. Brain Res 6:139-171.

Buzsáki G, Horvath Z, Urioste R, Hetke J, Wise K (1992) High frequency network oscillation in the hippocampus. Science 256:1025-1027.

Buzsáki G, Penttonen M, Nádasdy Z, Bragin A (1996) Pattern and inhibition-dependent invasion of pyramidal cell dendrites by fast spikes in the hippocampus in vivo. Proc Natl Acad Sci USA 93:9921-9925.

Callaway JC, Ross WN (1995) Frequency-dependent propagation of sodium action potentials in dendrites of hippocampal CA1 pyramidal neurons. J Neurophysiol 74:1-9.

Christie BR, Eliot LS, Ito K-I, Miyakawa H, Johnston D (1995) Different $\mathrm{Ca}^{2+}$ channels in soma and dendrites of hippocampal pyramidal neurons mediate spike-induced $\mathrm{Ca}^{2+}$ influx. J Neurophysiol 93:2553-2557.

Christie BR, Magee JC, Johnston D (1996) The role of dendritic action potentials and $\mathrm{Ca} 2+$ influx in the induction of homosynaptic long-term depression in hippocampal CA1 pyramidal neurons. Neurobiol Learn Mem 3:160-169.

Chrobak JJ, Buzsáki G (1996) High-frequency oscillations in the output networks of the hippocampal-entorhinal axis of the freely behaving rat. J Neurosci 16:3056-3066.

Csicsvari J, Hirase H, Moore K, Penttonen M, Buzsáki G (1997) Monosynaptic interactions between CA1 pyramidal cells and interneurons in the behaving rat. Soc Neurosci Absr 23:483.

Fox SE (1989) Membrane potential and impedance changes in hippocampal pyramidal cells during theta rhythm. Exp Brain Res 77:283-294.

Herreras O (1990) Propagation of dendritic action potential mediates synaptic transmission in CA1 pyramidal cells in situ. J Neurophysiol 64:1429-1441.

Hoffman DA, Magee JC, Colbert CM, Johnston D (1997) $\mathrm{K}^{+}$channel regulation of signal propagation in dendrites of hippocampal pyramidal neurons. Nature 387:869-875.

Hotson JR, Prince DA (1980) A calcium-activated hyperpolarization follows repetitive firing in hippocampal neurons. J Neurophysiol 43:409-419.

Ishizuka N, Cowan WM, Amaral DG (1995) A quantitative analysis of the dendritic organization of the pyramidal cells in the rat hippocampus. J Comp Neurol 362:17-45.

Jaffe DB, Johnston D, Lasser-Ross N, Lisman JE Miyakawa H, Ross WN (1992) The spread of $\mathrm{Na}^{+}$spikes determines the pattern of dendritic $\mathrm{Ca} 2+$ entry into hippocampal neurones. Nature $357: 244-246$.
Jaslove SW (1992) The integrative properties of spiny distal dendrites. Neuroscience 47:495-519.

Jester J, Campbell L, Sejnowski T (1996) Associative EPSP-spike potentiation induced by pairing orthodromic and antidromoc stimulation in rat hippocampal slices. J Physiol (Lond) 484:689-705.

Johnston D, Magee JC, Colbert CM, Christie BR (1996) Active properties of neuronal dendrites. Annu Rev Neurosci 19:165-186.

Jung HY, Mickus T, Spruston N (1997) Prolonged sodium channel inactivation contributes to dendritic action potential attenuation in hippocampal pyramidal neurons. J Neurosci 17:6639-6646.

Kim HG, Beierlein M, Connors BW (1995) Inhibitory control of excitable dendrites in neocortex. J Neurophysiol 74:1810-1814.

Leung LS, Yim CY (1986) Intracellular records of theta rhythm in hippocampal CA1 cells of the rat. Brain Res 367:323-327.

Llinás R, Nicholson C (1971) Electroresponsive properties of dendrites and somata in alligator Purkinje cells. J Neurophysiol 34:532-551.

Magee JC, Johnston D (1995) Synaptic activation of voltage-gated channels in the dendrites of hippocampal pyramidal neurons. Science 268:301-304.

Magee JC, Johnston D (1997) A synaptically controlled, associative signal for Hebbian plasticity in hippocampal neurons. Science 275:209-213.

Mainen ZF, Joerges J, Huguenard JR, Sejnowski TJ (1995) A model of spike initiation in neocortical pyramidal cells. Neuron 15:1427-1439.

Markram H, Lübke J, Frotscher M, Sakmann B (1997) Regulation of synaptic efficacy by coincidence of postsynaptic APs and EPSPs. Science 275:213-215.

Miles R, Tóth K, Gulyás AI, Hájos N, Freund TF (1996) Differences between somatic and dendritic inhibition in the hippocampus. Neuron $16: 815-823$

Miyakawa H, Ross WN, Jaffe D, Callaway JC, Lasser-Ross N, Lisman JE, Johnston D (1992) Synaptically activated increases in $\mathrm{Ca}^{2+}$ concentration in hippocampal CA1 pyramidal cells are primarily due to voltage-gated $\mathrm{Ca}^{2+}$ channels. Neuron 9:1163-1173.

Ranck Jr JB (1973) Studies on single neurons in dorsal hippocampal formation and septum in unrestrained rats. I. Behavioral correlates and firing repertoires. Exp Neurol 42:461-531.

Regehr W, Tank DW (1992) Calcium concentration dynamics produced by synaptic activation of CA1 pyramidal cell. J Neurosci 12:4202-4223.

Regehr W, Kehoe J, Ascher P, Amstrong C (1993) Synaptically triggered action potentials in dendrites. Neuron 11:145-151.

Schwartzkroin PA, Stafstrom CE (1980) Effect of EGTA on the calcium activated afterhyperpolarization in CA3 pyramidal cells. Science 210:1125-1126.

Segev I, Rall W (1988) Computational study of an excitable dendritic spine. J Neurophysiol 60:499-523.

Shepherd GM, Brayton RK (1987) Logic operations are properties of computer-simulated interactions between excitable dendritic spines. Neuroscience 21:151-166.

Sik A, Penttonen M, Ylinen A, Buzsáki G (1995) Hippocampal CA1 interneurons: an in vivo intracellular labeling study. J Neurosci 15:6651-6665.

Softky W (1994) Sub-millisecond coincidence detection in active dendritic trees. Neuroscience 58:13-41.

Soltész I, Deschénes M (1993) Low- and high-frequency membrane potential oscillations during theta activity in CA1 and CA3 pyramidal neurons of the rat hippocampus under ketamine-xylazine anesthesia J Neurophysiol 70:97-116.

Spencer WA, Kandel ER (1961) Electrophysiology of hippocampal neurons. IV. Fast prepotentials. J Neurophysiol 24:272-285.

Spruston N, Schiller Y, Stuart G, Sakmann B (1995) Activity dependent action potential invasion and calcium influx into hippocampal CA1 dendrites. Science 268:297-300.

Stuart G, Sakmann B (1994) Active propagation of somatic action potentials into neocortical pyramidal cell dendrites. Nature 367:69-72.

Stuart G, Spruston N, Sakmann B, Häuser M (1997) Action potential initiation and backpropagation in neurons of the mammalian CNS. Trends Neurosci 20:125-131.

Suzuki SS, Smith GK (1987) Spontaneous EEG spikes in the normal hippocampus. I. Behavioral correlates, laminar profiles and bilateral synchrony. Electroencephalogr Clin Neurophysiol 67:438-459.

Svoboda K, Denk W, Kleinfleld D, Tank DW (1997) In vivo dendritic 
calcium dynamics in neocortical pyramidal neurons. Nature 385:161-165.

Traub RD, Jefferys J, Miles R, Whittington M, Tóth K (1994) A branching dendritic model of a rodent CA3 pyramidal neurone. J Physiol (Lond) 481:79-95.

Tsubokawa H, Ross W (1996) IPSPs modulate spike backpropagation and associated $\left(\mathrm{Ca}^{2+}\right) \mathrm{i}$ changes in the dendrites of hippocampal CA1 pyramidal neurons. J Neurophysiol 76:2896-2906.

Turner RW, Meyers ER, Richardson TL, Barker JLJ (1991) The site for initiation of action potential discharge over the somatosensory axis of rat hippocampal CA1 neurons. J Neurosci 11:2270-2280.

Wong RKS, Stuart MJ (1992) Different firing patterns generated in dendrites and somata of CA1 pyramidal neurons in the guinea-pig hippocampus. J Physiol (Lond) 457:675-687.
Wong RKS, Prince DA, Basbaum AI (1979) Intradendritic recordings from hippocampal neurons. Proc Natl Acad Sci USA 76:986-990.

Ylinen A, Bragin A, Nádasdy Z, Jandó G, Szabó I, Sik A, Buzsáki G (1995a) Sharp wave associated high frequency oscillation $(200 \mathrm{~Hz})$ in the intact hippocampus: network and intracellular mechanisms. J Neurosci 15:30-46.

Ylinen A, Soltész I, Bragin A, Penttonen M, Sik A, Buzsáki G (1995b) Intracellular correlates of hippocampal theta rhythm in identified pyramidal cells, granule cells and basket cells. Hippocampus 5:78-90.

Yuste R, Denk W (1995) Dendritic spines as a basic unit of synaptic integration. Nature 375:682-684.

Yuste R, Tank D (1996) Dendritic integration in mammalian neurons, a century after Cajal. Neuron 16:701-716. 